\title{
Standard Model Particles with Mass Treated as Spheres with Finite Radii
}

\author{
T. R. Mongan \\ Sausalito, CA, USA \\ Email: tmongan@gmail.com
}

How to cite this paper: Mongan, T.R. (2020) Standard Model Particles with Mass Treated as Spheres with Finite Radii. Journal of Modern Physics, 11, 1993-1998. https://doi.org/10.4236/jmp.2020.1112126

Received: November 13, 2020 Accepted: December 18, 2020

Published: December 21, 2020

Copyright (c) 2020 by author(s) and Scientific Research Publishing Inc. This work is licensed under the Creative Commons Attribution International License (CC BY 4.0).

http://creativecommons.org/licenses/by/4.0/

\begin{abstract}
The particle physics Standard Model involves three charge 0 neutrinos, three charge $e$ leptons, three charge (2/3)e quarks, and three charge -(1/3)e quarks, where $e$ is electron charge. However, the Standard Model cannot explain why there are three generations of particles in each charge state and makes no predictions relating to quark and lepton masses. This analysis, treating Standard Model particles as spheres with radii 1/4 the particle Compton wavelength, explains three, and only three fermions are in each charge state and relates first generation quark masses to the electron mass.
\end{abstract}

\section{Keywords}

Standard Model Extension, Fermions Generations, Electron and First Generation Quark Masses

\section{Introduction}

The particle physics Standard Model cannot explain why there are three generations of quarks and lepton, and makes no predictions relating quark and lepton masses [1]. Furthermore, the Standard Model as presently constituted:

- must be modified to accommodate neutrino mass to account for oscillations between neutrino states as they propagate through space;

- treats fundamental particles as point particles with angular momentum $\hbar / 2$, or $\hbar$. Angular momentum is usually defined for rotating objects extended in space and, regarding point particles with angular momentum, we might ask what is rotating.

- is formulated in quantum field theory, based on continuum mathematics with infinite degrees of freedom. However, the holographic principle [2], developed from quantum mechanics, general relativity, and black hole thermodynamics, indicates only a large, but finite, number $\left[\sim 10^{122}\right]$ of bits of in- 
formation will ever be available to describe the observable universe, suggesting quantum field theory approximates an underlying discrete theory.

- involves point fermions with infinite energy density and physical theories involving infinities are logically complicated and debatable [3].

This analysis, treating Standard Model particles with mass as spheres with finite radii, addresses these important issues. In particular, it explains why three, and only three fermions are in each charge state and relates first generation quark masses to electron mass.

\section{Standard Model Particle Masses}

Masses $m_{i}$, in $\left(\mathrm{MeV} / \mathrm{c}^{2}\right)$, of charged Standard Model fermions [4] are in Table 1. Compton wavelengths are $l_{i}=\hbar c / m_{i}$, with $\hbar$ Planck's constant, $c=3 \times 10^{10} \mathrm{~cm} / \mathrm{sec}$ the speed of light, $\hbar c=197.32 \mathrm{MeV} \cdot \mathrm{F}, 1 \mathrm{~F}=1 \times 10^{-13} \mathrm{~cm}$, and charges in fractions of electron charge $e$.

Neutrino masses are estimated using known [5] quantities

$$
a^{2}=m_{2}^{2}-m_{1}^{2}=7.37 \times 10^{-5}(\mathrm{eV})^{2} \text { and } b^{2}=m_{3}^{2}-\frac{1}{2}\left(m_{1}^{2}+m_{2}^{2}\right)=2.50 \times 10^{-3}(\mathrm{eV})^{2} \text {, }
$$
assuming neutrino mass sum $\Sigma_{v}$ equals upper bound [6] $\Sigma_{v}=0.12 \mathrm{eV}$. $\Sigma_{v}=\sqrt{m_{2}^{2}-a^{2}}+m_{2}+\sqrt{m_{3}^{2}+b^{2}-\left(a^{2} / 2\right)}$, solved by successive approximation, results in $m_{1}=0.030 \mathrm{eV}, m_{2}=0.031 \mathrm{eV}$, and $m_{3}=0.059 \mathrm{eV}$. Massive fundamental bosons are $\mathrm{W}^{ \pm}$with mass $80.4 \mathrm{GeV} / \mathrm{c}^{2}, Z$ with mass $91.2 \mathrm{GeV} / \mathrm{c}^{2}$, and Higgs with mass $125 \mathrm{GeV} / \mathrm{c}^{2}$.

\section{Treating Fundamental Fermions as Godel Systems}

This analysis describes fundamental fermions as spherical shells with radius $\frac{l}{4}$ rotating around an axial core centered on the axis of rotation, with half of any fermion charge on the shell surface at each end of the axis of rotation. Fundamental fermions are then represented by Godel solutions of Einstein's equations, with average matter density $\rho$ equal average fermion mass density, pressure

Table 1. Mass, wavelengths, and charge of charged standard model fermions.

\begin{tabular}{cccc}
\hline Fermion & Mass $\boldsymbol{m}_{\boldsymbol{i}}\left(\mathrm{MeV} / \mathrm{c}^{2}\right)$ & Wavelength $\boldsymbol{I}_{\boldsymbol{i}}(\mathrm{F})$ & Charge \\
\hline Electron & 0.511 & 386 & $e$ \\
Up quark & 2.16 & 91.4 & $2 e / 3$ \\
Down quark & 4.67 & 42.3 & $-e / 3$ \\
Strange quark & 93 & 2.12 & $-e / 3$ \\
Muon & 105.7 & 1.87 & $e$ \\
Charm quark & 1270 & 0.155 & $e e / 3$ \\
Tau lepton & 1777 & 0.111 & $-e / 3$ \\
Bottom quark & 4180 & 0.0472 & $2 e / 3$ \\
Top quark & 173,000 & 0.00114 & \\
\hline
\end{tabular}


$\left(\frac{1}{2}\right) \rho c^{2}$ from negative vacuum energy density $-\left(\frac{1}{2}\right) \rho c^{2}$, and effective internal gravitational constant $G_{f}$ determined by $\omega=2 \sqrt{\pi G_{f} \rho}$. Charge is on the shell at the axis of rotation, so rotation does not cause radiative loss of energy by accelerated charge. Rotation axis orientation is unknown until $z$ component of fermion angular momentum is measured, so fermion mass appears sinusoidally distributed on a disk of radius (1/4) perpendicular to the line of sight.

\section{Mass and Pressure Distribution in Fundamental Fermions Allow only Three Fermions in Each Charge State}

Fundamental fermions, considered as spheres with size characterized by Compton wavelengths $l$, have three associated geometric quantities, volume $\sim \beta$, surface area $\sim P$, and diameter $\sim l$. Mass and pressure distribution in fundamental fermions identifies three wavelengths in each charge state as solutions of a cubic equation $\mathrm{Al}^{3}+\mathrm{Bl}^{2}+\mathrm{Cl}=0$. Describing mass and pressure distribution in terms of surface and linear elements requires minimum shell thickness and core radius near the Planck length $l_{P}=\sqrt{\frac{\hbar G}{c^{3}}}$. In each charge state $\frac{n e}{3}$, with $n=0,1,2$ or 3 , total fermion mass is the sum of mass equivalent of pressure, $\frac{m}{2}$, in the volume, mass equivalent of surface pressure $\frac{\pi}{4} S l^{2}$, and core mass $L l$. So $\frac{4}{3} \pi \rho\left(\frac{l}{4}\right)^{3}=\frac{4}{3} \pi \frac{\rho}{2}\left(\frac{l}{4}\right)^{3}+4 \pi S\left(\frac{l}{4}\right)^{2}+2 L\left(\frac{l}{2}\right)$. Written as $A l^{3}-B l^{2}-C l=0$, with $A=\frac{\pi}{96} \rho, B=\frac{\pi S}{4}$, and $C=2 L$, the discriminant $B^{2} C^{2}-4 A C^{3}$ is positive regardless of the sign of $B$ and the equation has three real roots corresponding to three fermion Compton wavelengths in a charge state. Nickalls [7] showed wavelengths $l=\frac{\hbar}{m c}$ satisfying the equation correspond to projections on the laxis, defined by an angle $\Theta$, of vertices of an equilateral triangle. $\Theta$ is the angle between two lines starting at the center of the triangle, one parallel to the $I$ axis and one extending to the rightmost vertex of the equilateral triangle. Nickall's parameters $l_{N}=-\frac{B}{3 A}=-\frac{8 S}{\rho}, \quad \delta^{2}=l_{N}^{2}-\frac{C}{3 A}=l_{N}^{2}-\frac{64 L}{\rho}, \quad 3 l_{N}=l_{1}+l_{2}+l_{3}$, and $\delta^{2}=\frac{\left(l_{1}-l_{N}\right)^{2}}{4}+\frac{\left(l_{2}-l_{3}\right)^{2}}{12}$, identify roots $l_{1}=l_{N}+2 \delta \cos \Theta$,

$l_{2}=l_{N}-\delta(\cos \Theta-\sqrt{3} \sin \Theta)$, and $l_{3}=l_{N}-\delta(\cos \Theta+\sqrt{3} \sin \Theta)$ corresponding to fermion Compton wavelengths in a charge state. Three positive Compton wavelengths in each charge state require negative surface mass equivalent density $S=-\frac{\rho l_{N}}{8}, \delta^{2}<l_{N}^{2}$ in each charge state, and positive mass per unit core length $L=\frac{\rho}{64}\left(l_{N}^{2}-\delta^{2}\right)$. Negative $S$ results from positive shell vacuum energy density 
$\left(\frac{1}{2}\right) \rho c^{2}$, opposite the negative vacuum energy density $-\left(\frac{1}{2}\right) \rho c^{2}$ in the volume. Then negative pressure equivalent mass inside the shell counters positive pressure equivalent mass in the volume. With no net pressure at the fermion surface, no force acts to increase or decrease fermion size, necessary for stable fundamental fermions as Godel solutions within our universe. Total fundamental fermion mass $=\frac{\pi}{48} \rho l^{3}$, surface mass $=-\frac{\pi}{32} \rho l_{N} l^{2}$, and core mass $=\frac{\pi}{64} l\left(l_{N}^{2}-\delta^{2}\right)$, so surface mass multiple of total mass $=-\frac{3}{2} \frac{l_{N}}{l}$ and core mass multiple of total mass $=$ $\frac{3}{4} \frac{\left(l_{N}^{2}-\delta^{2}\right)}{l^{2}}$. Surface mass and core mass multiple increase with decreasing $I$ because smaller fermion volume at smaller $l$ decreases volume occupied by positive pressure from negative vacuum energy density of Godel solutions, while $($ surface mass $) /($ core mass $)=-\frac{2 l_{N} l}{\left(l_{N}^{2}-\delta^{2}\right)}$ increases with $l$.

\section{Angular Velocity and Internal Gravitational Constant from Angular Momentum $\hbar / 2$}

Fermion spheres with radius $\frac{l}{4}$ and core radius $r$ have moment of inertia $I=\frac{2}{5} \frac{m}{2}\left(\frac{l}{4}\right)^{2}+\frac{2}{3} \frac{\pi}{4} S l^{2}\left(\frac{l}{4}\right)^{2}+\frac{1}{2} L l r^{2}$, with negligible last term because $r \ll l$. Angular velocity $\omega=\frac{\hbar}{2 I}=\frac{8 c}{\left(0.2 l-l_{N}\right)}$ and tangential speed of points on the spherical shell equator as multiple of the speed of light $\frac{v_{T}}{c}=\frac{\omega l}{4 c}=\frac{2 l}{\left(0.2 l-l_{N}\right)}$. $\frac{v_{T}}{c}>1$ for lowest mass fermions in each charge state, with closed time-like curves within those Godel solutions acceptable in fundamental fermions unchanging from creation to annihilation. From $\omega=2 \sqrt{\pi G_{f} \rho}, \frac{G_{f}}{G}=\frac{3 l^{4}}{l_{P}^{2}\left(0.2 l-l_{N}\right)^{2}}$.

\section{Charge and Ground State Fundamental Fermion Mass}

Ground state fundamental fermions constituents of atoms and molecules, differ from other generation fundamental fermions by having core mass less than total mass, tangential speeds $\frac{V_{T}}{c}>1$, and larger internal gravitational constants. With fine structure constant $\frac{e^{2}}{\hbar c}=\frac{1}{137}$, electrostatic potential energy of fundamental fermions from repulsion between equal surface charges at the rotation axis is $\left(\frac{n e}{6}\right)^{2} /\left(\frac{l}{2}\right)=n^{2} \frac{m e^{2}}{9 \hbar c}=n^{2} \frac{m}{1233}$. If electrostatic potential energy is the same for 
all charged ground state fundamental fermions and electron mass $m_{e}=0.511 \mathrm{MeV}$, up quark mass $m_{u}=4 m_{e}=2.04 \mathrm{MeV}$ and down quark mass $m_{d}=9 m_{e}=4.60 \mathrm{MeV}$, well within quark mass error bars [4]. All charged fundamental fermion masses and charges can be related to election charge and mass.

\section{Massive Standard Model Bosons as Spheres with Finite Radii}

Treating massive Standard Model bosons as spheres with finite radii and an internal gravitational constant is simpler than for fundamental fermions. $\mathrm{W}^{ \pm}$and $Z$ bosons as uniform spheres with moment of inertia $I=\frac{2}{5} m r^{2}$ and radius $r=\frac{l}{4}$ have angular momentum $\hbar=I \omega$, so $\omega=\frac{40 \hbar}{m l^{2}}$. For Godel systems with internal gravitational constant $G_{i}, \omega=2 \sqrt{\pi G_{i} \rho}$, resulting in $\frac{G_{i}}{G}=\frac{25}{3}\left(\frac{l}{l_{P}}\right)^{2}$, where Planck length $l_{P}=1.62 \times 10^{-20} \mathrm{~F}$. Higgs bosons are treated as static Einstein solutions of general relativity with matter energy density $\rho c^{2}$ and positive vacuum energy density $\frac{1}{2} \rho c^{2}$, opposite the negative vacuum energy density of Godel solutions. The Friedmann equation for the radius of those closed, homogeneous, isotropic systems with internal gravitational constant $G_{i}$ is $\left(\frac{\mathrm{d} R}{\mathrm{~d} t}\right)^{2}-\frac{8 \pi G_{i}}{3}\left[\rho c^{2}\left(\frac{R_{0}}{R}\right)^{3}+\frac{1}{2} \rho c^{2}\right]\left(\frac{R}{c}\right)^{2}=-c^{2}$, with $\left(\frac{\mathrm{d} R}{\mathrm{~d} t}\right)=0, R=R_{0}=\frac{l_{H}}{4}$, and Higgs Compton wavelength $l_{H}$, resulting in $\frac{G_{i}}{G}=\frac{1}{12}\left(\frac{l_{H}}{l_{P}}\right)^{2}$.

\section{Conclusions}

Considering fundamental fermions as spherical shells rotating around an axial core, identified with Godel solutions to Einstein's equations, in each charge state,

- three fermion wavelengths specify volume, surface, and core mass as multiples of total fermion mass, and

- each fermion mass and wavelength specify angular velocity, tangential speed of points on the shell equator, and effective internal fermion gravitational constant.

Electrostatic potential energy in ground state fundamental fermions, from repulsion between equal surface charges at the rotation axis, is identical if up quark mass $=4 m_{e}=2.04\left(\mathrm{MeV} / \mathrm{c}^{2}\right)$ and down quark mass $=9 m_{e}=4.60\left(\mathrm{MeV} / \mathrm{c}^{2}\right)$.

\section{Acknowledgements}

Inquiry on the nature of mass by the late UCSF Professor Leon Kaufman, and on the nature of spin by my wife, Lou Mongan, led to this analysis. 


\section{Conflicts of Interest}

The author declares no conflicts of interest regarding the publication of this paper.

\section{References}

[1] https://home.cern/science/physics/standard-model

[2] Bousso, R. (2002) Reviews of Modern Physics, 74, 825. arXiv:hep-th/0203101 https://doi.org/10.1103/RevModPhys.74.825

[3] Hilbert, D. (1926) Mathematische Annalen (Berlin), 95, 161. Ellis, G., Meissner, K. and Nicolai, H. (2018) Nature Physics, 14, 770. https://doi.org/10.1038/s41567-018-0238-1

[4] Zyla, P., et al., (Particle Data Group) (2020) Prog. Theor. Exp. Phys., 2020, 083 C01.

[5] Capozzi, S., Lisi, E., Marrone, A., Montanino, D. and Palazzo, A. (2016) Nuclear Physics B, 908, 1-14. [arXiv:1601.07777] https://doi.org/10.1016/j.nuclphysb.2016.02.016

[6] Vagnozzi, S. (2109) Cosmological Searches for the Neutrino Mass Scale and Mass Ordering. Stockholm University PhD Thesis. [arXiv:1907.08010]

[7] Nickalls, R. (1993) The Mathematical Gazette, 77, 354-359. https://doi.org/10.2307/3619777 